\title{
Quantification of the heterogeneity of particle packings
}

\author{
Iwan Schenker, ${ }^{*}$ Frank T. Filser, and Ludwig J. Gauckler \\ Department of Materials, Nonmetallic Materials, ETH Zurich, Zurich CH-8093, Switzerland
}

Tomaso Aste

Department of Applied Mathematics, RSPhysSE, The Australian National University, 0200, Australia and School of Physical Sciences, University of Kent, Canterbury, Kent CT2 7NH, United Kingdom

Hans J. Herrmann

Computational Physics for Engineering Materials, Institute for Building Materials, ETH Zurich, Zurich CH-8093, Switzerland

(Received 19 January 2009; revised manuscript received 12 May 2009; published 5 August 2009)

\begin{abstract}
The microstructure of coagulated colloidal particles, for which the interparticle potential is described by the Derjaguin-Landau-Verweg-Overbeek theory, is strongly influenced by the particles' surface potential. Depending on its value, the resulting microstructures are either more "homogeneous" or more "heterogeneous," at equal volume fractions. An adequate quantification of a structure's degree of heterogeneity (DOH), however, does not yet exist. In this work, methods to quantify and thus classify the DOH of microstructures are investigated and compared. Three methods are evaluated using particle packings generated by Brownian dynamics simulations: (1) the pore size distribution, (2) the density-fluctuation method, and (3) the Voronoi volume distribution. Each method provides a scalar measure, either via a parameter in a fit function or an integral, which correlates with the heterogeneity of the microstructure and which thus allows to quantitatively capture the DOH of a granular material. An analysis of the differences in the density fluctuations between two structures additionally allows for a detailed determination of the length scale on which differences in heterogeneity are most pronounced.
\end{abstract}

DOI: 10.1103/PhysRevE.80.021302

PACS number(s): 81.05.Rm, 61.43.- j, 82.70.Gg

\section{INTRODUCTION}

Colloidal particle packings are suitable model systems for the study of the structural properties of granular materials below the random loose packing limit. For such systems, the gravitational force is negligible in comparison to the van der Waals force, electrostatic repulsion, or Brownian motion $[1,2]$. In the present study, we particularly focus on systems, for which the local arrangement of the particles is the only variable, as opposed to variations in the volume fraction or the particle size distribution, for example. Commonly, these microstructures are referred to as more homogeneous or more heterogeneous, which either designates a structure presenting a rather uniform distribution of the particle positions or one having locally denser regions and therefore larger voids. These qualitative terms may be intuitive; however, they do not allow for a sound scientific quantification of the structure's degree of heterogeneity (DOH), which does not yet exist. In this paper, three methods providing the means for such a quantification are presented, analyzed, and compared. These methods permit to explicitly capture the DOH of a particle packing in form of a quantitative scalar measure.

Experimentally, the reproducible generation of colloidal particle packings possessing a specific $\mathrm{DOH}$ is achieved by the use of an in situ enzyme-catalyzed destabilization method (direct coagulation casting (DCC) $[3,4]$ ). For volume fractions between 0.2 and 0.6, DCC allows for the coagulation of electrostatically stabilized colloidal suspensions to

\footnotetext{
*iwan.schenker@mat.ethz.ch
}

stiff particle structures by either shifting the $p \mathrm{H}$ of the suspension to the particles' isoelectric point or by increasing the ionic strength of the suspension without disturbing the particle system. Shifting the $p \mathrm{H}$ leads to a more homogeneous microstructure through diffusion-limited aggregation, while increasing the ionic strength results in more heterogeneous microstructures via the reaction-rate-limited aggregation. The differences in heterogeneity have been observed using various techniques such as diffusing wave spectroscopy [5], static light transmission [5], or cryogenic scanning electron microscopy [6].

Computationally, microstructures with different $\mathrm{DOH}$ were successfully reproduced using Brownian dynamics (BD) simulations [6-8]. Slices of three particle layer thickness through a homogeneous and a heterogeneous BD microstructure of an identical volume fraction nicely demonstrate the differences between the microstructures (Fig. 1). The microstructure on the left presents a rather uniform distribution of the particle positions over the whole slice, while the microstructure on the right presents locally more densely packed particles and, consequently, larger voids. Both structures have an identical overall volume fraction of 0.4.

In preceding works, various characterization methods, such as the radial pair-correlation function [7], the bond angle distribution function [7], the triangle distribution function [7], and the Minkowski functionals in conjunction with the parallel-body technique [9], were applied to sets of microstructures generated by BD simulations [8].

The pair-correlation function quantifies the amount of structural rearrangement during the coagulation. Its usefulness regarding a distinction between structures with different $\mathrm{DOH}$, however, is rather limited as the differences between 

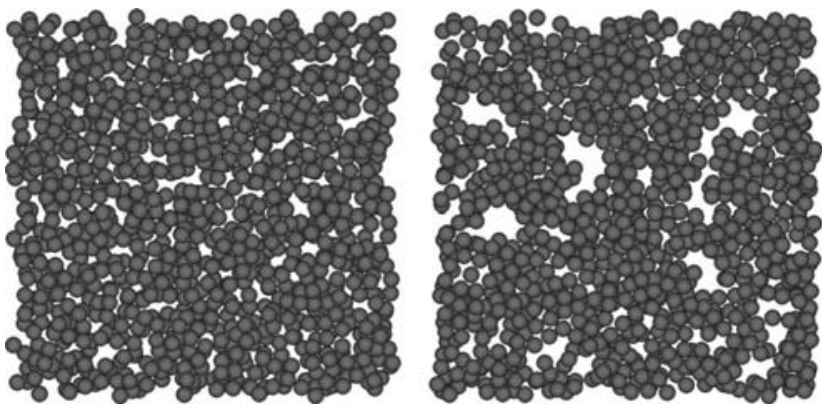

FIG. 1. Slices through a homogeneous (left) and a heterogeneous (right) particle structure with the same volume fraction of 0.4 resulting from Brownian dynamics simulations [7] (slice thickness: three particle diameters; particle diameter: $0.5 \mu \mathrm{m}$ ).

peaks corresponding to characteristic particle separation distances are relatively small [7]. The main advantage of the pair-correlation function is its experimental accessibility through scattering techniques such as spin-echo small-angle neutron scattering (SESANS) [10].

The bond angle distribution function and the triangle distribution function give further information on the local building blocks of the particle network [7]. Particular features, as, for example, peaks in the respective distribution function, allow distinguishing between more homogeneous and more heterogeneous microstructures. However, as in the case of the pair-correlation function, the differences between structures with different DOH are small for both the bond angle and the triangle distribution function.

The analysis using the Minkowski functionals in conjunction with the parallel-body technique supplies additional information on the structure's morphology resolving microstructural differences on a length scale limited by the largest pore size [9]. This method is computationally intensive and the extension to arbitrary particle shapes is difficult.

Gearing toward a possible correlation between microstructure and mechanical properties homogeneous and heterogeneous microstructures have recently been analyzed in terms of load bearing substructures: first, regions of closely packed particles and, second, quasilinear chains of contacting particles [11]. The locally closed packed regions were analyzed using the common neighbor distribution in conjunction with the dihedral angle distribution. Both methods only showed minor differences between a homogeneous and a heterogeneous microstructure. In particular, practically the same number of triangles and regular tetrahedrons was found in both structures. Quasilinear arrangements of contacting particles were quantified using the straight path method, revealing significant microstructural differences between homogeneous and heterogeneous. Approximately, twice as many paths of length longer or equal to four particles and three times as many paths of length longer or equal to five particles were found in the heterogeneous microstructure.

Despite the multitude of microstructural characterization methods that has been applied to colloidal microstructures possessing various $\mathrm{DOH}$, a useful quantification of the microstructures' heterogeneity in the form of a scalar measure is lacking. In the present study, statistical measures allowing for a quantification of a structure's heterogeneity are provided. The following three methods aiming at this quantification are discussed.

First, the exclusion probability [12] estimates the pore size distribution by randomly probing the pore space. In [13], this method was applied to very dilute simulated colloidal systems and allowed for a clear distinction between particle gel networks with varying textures. The same method termed as spherical contact distribution function [14] was used to investigate the pore size distribution of dense sphere packings as a function of the particle size distribution and the packing generation algorithm.

Second, the density-fluctuation method considers the fixed particle centers as a point process, and it statistically analyzes the fluctuations of the particle center density as a function of length scale. Used comparatively, this method further allows for a detailed analysis of the length scale, on which two structures present the largest differences in terms of heterogeneity.

Finally, the Voronoi volume distribution [15] is used to quantify the distribution of the free volume of our particle packings. In [16], the packing of cohesive particles resulting from simulations using the discrete element method [17] with volume fractions between approximately 0.2 and 0.6 was analyzed. The distribution of Voronoi volumes was shown to broaden with decreasing volume fraction. In [18], the Voronoi volume distribution was determined for a large set of experimental and numerical data covering a wide range of volume fractions. The various distributions were shown to follow a so-called $k$-gamma function, which was deduced by means of a statistical-mechanics approach. The parameter $k$ characterizing the shape of the curve was found to depend very sensitively on the structural organization of the particles.

To the authors' knowledge, none of these methods have yet been applied to particle structures, for which the DOH is the only variable, in opposition to a varying volume fraction or particle size distribution, for example.

\section{MATERIALS AND METHODS}

In the following, the structure characterization methods employed in this work are presented: the pore size distribution, the density-fluctuation method, and the distribution of Voronoi volumes. These methods are evaluated in terms of their ability to quantify the DOH of microstructures generated by previous $\mathrm{BD}$ simulations $[7,8]$. In these simulations, the Derjaguin-Landau-Verweg-Overbeek (DLVO) theory [19] was used to describe the interparticle potential $V^{D L V O}$ given by the sum of an attractive van der Waals term $V^{v d w}$ [Eq. (1)] and an electrostatic repulsion term $V^{e l}$ [Eq. (2)]. Thus, $V^{D L V O}=V^{v d w}+V^{e l}$ with

$$
V^{v d w}(r)=\frac{-A_{H}}{12}\left[\frac{d_{0}^{2}}{r^{2}-d_{0}^{2}}+\frac{d_{0}^{2}}{r^{2}}+2 \ln \left(\frac{r^{2}-d_{0}^{2}}{r^{2}}\right)\right],
$$


TABLE I. DLVO potential parameters.

\begin{tabular}{lcc}
\hline \hline Parameter & Symbol & Value \\
\hline Hamaker constant of $\mathrm{Al}_{2} \mathrm{O}_{3}$ in $\mathrm{H}_{2} \mathrm{O}$ & $A_{H}$ & $4.76 \times 10^{-20} \mathrm{~J}$ \\
Particle diameter & $d_{0}$ & $5 \times 10^{-7} \mathrm{~m}$ \\
Relative dielectric constant of $\mathrm{H}_{2} \mathrm{O}$ & $\epsilon_{r}$ & 81 \\
Surface potential & $\Psi_{0}$ & $0-15 \mathrm{mV}$ \\
Absolute temperature & $T$ & $293 \mathrm{~K}$ \\
Valency of ions & $z$ & 1 \\
Inverse Debye screening length & $\kappa$ & $10^{8} \mathrm{~m}^{-1}$ \\
\hline
\end{tabular}

$$
V^{e l}(r)=\pi \epsilon_{r} \epsilon_{0}\left[\frac{4 k_{b} T}{z e} \tanh \left(\frac{z e}{4 k_{b} T} \Psi_{0}\right)\right]^{2} d_{0} \exp \left(-\kappa\left\{r-d_{0}\right\}\right),
$$

respectively. The DLVO parameters are summarized in Table I.

The heterogeneity of the final microstructure was shown to be closely related to the presence and depth of the secondary minimum in the interparticle potential, which, for fixed values of the Debye screening length, essentially depends on the particles' surface potential $\Psi_{0}[7,20]$.

The microstructures analyzed in this work are labeled according to the surface potential $\Psi_{0}$ used during their generation. In particular, the following $\Psi_{0}$ values are used: 0,12 , 13, 14, and $15 \mathrm{mV}$. Additionally, these final microstructures are compared to the initial microstructure, representing a stabilized suspension in which the interparticle potential is purely repulsive. The volume fraction is fixed at 0.4 , the monosized particles have a radius $r_{0}=0.25 \mu \mathrm{m}$, all microstructures consist of 8000 particles and are contained in a simulation box with periodic boundary conditions. In particular, the particle interpenetration is much smaller than the length scale of the heterogeneities analyzed in this study. Less than $0.1 \%$ of all contacts present an interpenetration above $1.0 \%$ of the particle diameter $d_{0}$ with a maximum interpenetration of $1.3 \% d_{0}$. Please refer to $[7,8]$ for a more detailed description of the BD simulations.

\section{A. Pore size distribution}

The pore size distribution is estimated following the approach described by Torquato et al. [12] using the exclusion probability $E_{V}(r) . E_{V}(r)$ is defined as the probability of inserting a "test" particle of radius $r$ at some arbitrary position in the pore space of a system of $N$ particles. This is schematically represented in Fig. 2 using a set of particles of radius $r_{0}$ (gray) with a test particle of radius $r$ inserted at position $P$.

In order to estimate $E_{V}(r)$, a statistically large number of points is randomly placed in the pore space of a given microstructure and the distance to the closest particle surface is determined.

In [9], the relation between $E_{V}(r)$ and the Minkowski functional $W_{1}(r)$ was described. The Minkowski functional in conjunction with the parallel-body technique considers the point process given by the fixed particle centers. Generally,

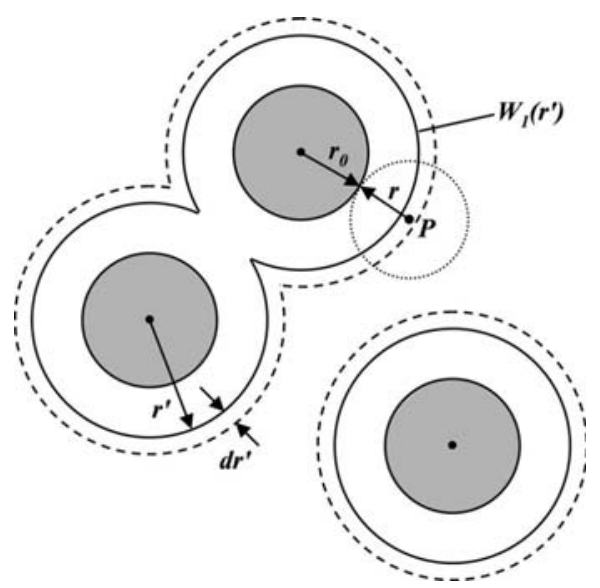

FIG. 2. Particle structure (gray particles) with a test particle inserted at position $P$.

in three dimensions there are four functionals $W_{i}$, where $i$ $=0, \ldots, d$ with $d$ as the spatial dimension, corresponding to the volume, surface, average mean curvatures, and connectivity. In particular, $W_{1}\left(r^{\prime}\right)=\frac{1}{3} \int_{\partial A\left(r^{\prime}\right)} d S$ calculates the surface of the union of spheres located at the particle centers in dependence of their radius $r^{\prime}=r_{0}+r$. Schematically, $W_{1}\left(r^{\prime}\right)$ is shown in Fig. 2. $W_{1}\left(r^{\prime}\right) d r^{\prime}$ is the volume between the distance $r^{\prime}$ and $r^{\prime}+d r^{\prime}$. The probability of placing an uniformly drawn random test point at a distance $r \in\left[r^{\prime}, r^{\prime}+d r^{\prime}[\right.$ is proportional to the volume delimited by $r^{\prime}$ and $r^{\prime}+d r^{\prime}$ and, therefore, $\quad P\left(r^{\prime} \leq r_{0}+r<r^{\prime}+d r^{\prime}\right)=E_{V}(r) d r^{\prime} \propto W_{1}\left(r^{\prime}\right) d r^{\prime}$, which results in $E_{V}\left(r_{0}+r\right) \propto W_{1}\left(r^{\prime}\right)$. Thus, given a statistically large number of test points $E_{V}(r)$ provides a means to estimate $W_{1}\left(r^{\prime}\right)$, which has the advantage of being computationally less intensive. A similar Monte Carlo integration is usually performed to calculate $W_{0}\left(r^{\prime}\right)$.

\section{B. Density fluctuations}

The density-fluctuation method quantifies the local fluctuation of particle centers by subdividing the structure into smaller parts and measuring the average value and the standard deviation of the particle center density. Practically, this is done by dividing the structure into $n_{c}^{3}$ cells using a cubic grid, where $n_{c}$ is the number of cells along one dimension with $n_{c}=2, \ldots, n_{c}^{\max }, n_{c}^{\max }$ being the maximum number of cells under consideration (along one dimension). The density-fluctuation method consists in determining the average number of particle centers per cell $E_{p p c}$ and its standard deviation $\sigma_{p p c}$ as a function of $n_{c}$ and then calculating the relative fluctuations $\frac{\sigma_{p p c}}{E_{p p c}}$.

Additionally, the density-fluctuation method is applied comparatively allowing for a determination of the length scale on which two structures show the largest differences. Therefore, the difference $\Delta\left(n_{c}\right)$ given in Eq. (3) is calculated between two structures $i$ and $j$,

$$
\Delta\left(n_{c}\right)=\left[\frac{\sigma_{p p c}^{i}}{E_{p p c}^{i}}-\frac{\sigma_{p p c}^{j}}{E_{p p c}^{j}}\right]\left(n_{c}\right) .
$$


If both structures have the same number of particles and identical volume fractions, which is the case for the microstructures investigated in this work, then $E_{p p c}^{i}=E_{p p c}^{j}=E_{p p c}$ and Eq. (3) yields

$$
\Delta\left(n_{c}\right)=\frac{\sigma_{p p c}^{i}-\sigma_{p p c}^{j}}{E_{p p c}}\left(n_{c}\right) .
$$

$\Delta$ is plotted against the cell's edge length $l_{c}\left(n_{c}\right)=L_{b o x} / n_{c}$ normalized by the particle diameter $d_{0}$, where $L_{b o x}$ is the side length of the cubic simulation box.

\section{Voronoi volume distribution}

Formally, for a set of monodispersed spherical particles, the Voronoi volume $V_{i}$ associated with a particle $i$ is a polyhedron whose interior consists of all points in space that are closer to the center of particle $i$ than to any other particle center [15]. The Voronoi tessellation thus divides the volume containing a set of particles into a set of space-filling, nonoverlapping, and convex polyhedrons. In this work, the Quickhull algorithm [21] is used to compute the volumes of the Voronoi polyhedrons. The distribution of the Voronoi volumes describes the deviation of a structure from a perfect crystalline packing, in which case all particles occupy the same volume and the Voronoi volume distribution thus is a delta function. The minimum volume of a Voronoi cell $V_{\text {min }}$ is achieved for a regular close packing with $V_{\text {min }}$ $=1.325 V_{\text {sphere }}$, where $V_{\text {sphere }}$ is the volume of a particle. The difference between a particle's Voronoi volume $V_{i}$ and $V_{\text {min }}$ is termed as the Voronoi free volume $V_{i}^{f}=V_{i}-V_{\text {min }}$.

The distribution of the Voronoi free volume was found to follow gamma distributions: Kumar and Kumaran, for example, showed that the free volume distribution of hard-disk and hard-sphere systems are well described using a twoparameter and a three-parameter gamma distributions [22].

Aste and Di Matteo deduced the two-parameter gamma distribution using a statistical-mechanics approach [18]. The so-called $k$-gamma distribution given by Eq. (5) was found to agree very well with a large number of experiments and computer simulations over a wide range of packing fractions,

$$
f\left(V^{f}, k\right)=\frac{k^{k}}{\Gamma(k)} \frac{\left(V^{f}\right)^{k-1}}{\left(\bar{V}^{f}\right)^{k}} \exp \left(-k \frac{V^{f}}{\bar{V}^{f}}\right) .
$$

The mean Voronoi free volume $\bar{V}^{f}$ is a scaling parameter given by $\bar{V}-V_{\min }=(1 / \Phi-1.325) V_{\text {sphere }}$, where $\Phi$ is the volume fraction. The free parameter $k$ characterizing the shape of the curve depends very sensitively on the structural organization of the particles and corresponds to the specific heat in classical thermodynamics. Empirically, $k$ can be computed using $k=\frac{(\bar{V} f)^{2}}{\sigma_{V}^{2}}$, where $\sigma_{V}^{2}$ is the variance of the free volume distribution. In particular, Eq. (5) was shown to hold for systems at statistical equilibrium as well as for systems out of equilibrium [18].

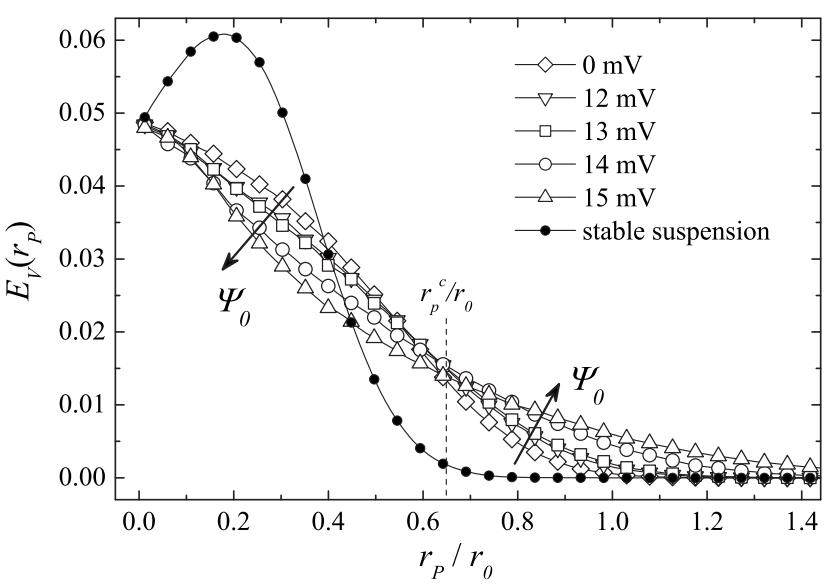

FIG. 3. Pore size distribution for the initial microstructure (stable suspension) and final microstructures $\left(\Psi_{0}=0-15 \mathrm{mV}\right)$. $B$-spline curves serve as guide for the eyes.

\section{RESULTS AND DISCUSSION}

\section{A. Pore size distribution}

The pore size distribution of the various microstructures is depicted in Fig. 3 in terms of the exclusion probability $E_{V}\left(r_{P}\right)$ as a function of the pore radius $r_{P}$ normalized by the particle radius $r_{0}$. A set of $10^{6}$ random test points [23] placed in the structures' pore space was used to estimate $E_{V}\left(r_{P}\right)$.

All curves for the final microstructures $\left(\Psi_{0}=0-15 \mathrm{mV}\right)$ decrease monotonically toward increasing pore sizes, indicating a decreasing probability of finding larger pores. A particular behavior is found for the stable suspension, where the exclusion probability increases with increasing pore size up to a pore radius of $0.18 r_{0}$. This is due to the repulsive interparticle potential in the case of the stable suspension where, consequently, the particles are not in contact. The pore diameter, at which the maximum in the exclusion probability is found, corresponds to the average surface-tosurface distance between neighboring particles.

Remarkably, the various curves for the final microstructures in Fig. 3 intersect at approximately the same point defining a characteristic pore size $r_{p}^{c}$ found at $0.65 r_{0}$. The probability of finding a pore with a radius below $r_{p}^{c}$ decreases for increasing values of $\Psi_{0}$, while pores with a radius above $r_{p}^{c}$ are found with a higher probability toward increasing $\Psi_{0}^{p}$. Indeed, the probability of finding pore radii larger than approximately $1.1 r_{0}$ is negligible in the case of the most homogeneous microstructure with $\Psi_{0}=0 \mathrm{mV}$, while pore radii up to $2.4 r_{0}$ are found in the most heterogeneous microstructure with $\Psi_{0}=15 \mathrm{mV}$.

Using the results obtained for the exclusion probability $E_{V}\left(r_{P}\right)$, the cumulative probability $P\left(r_{P}>r\right)$ of finding pore radii larger than $r$ was calculated using $P\left(r_{P}>r\right)$ $=\Sigma_{r^{\prime}>r_{P}} E_{V}\left(r^{\prime}\right)$. The results are shown in Fig. 4.

$P\left(r_{P}>r\right)$ decreases monotonically for all microstructures. The fastest decrease is found for the stable suspension. With increasing $\Psi_{0}$, the decrease of $P\left(r_{P}>r\right)$ is slower. Comparing the "most and the least heterogeneous" microstructure, with $\Psi_{0}=15 \mathrm{mV}$ and $\Psi_{0}=0 \mathrm{mV}$, respectively, there is a 1.7 times higher probability of finding pores larger than $0.5 r_{0}$. 


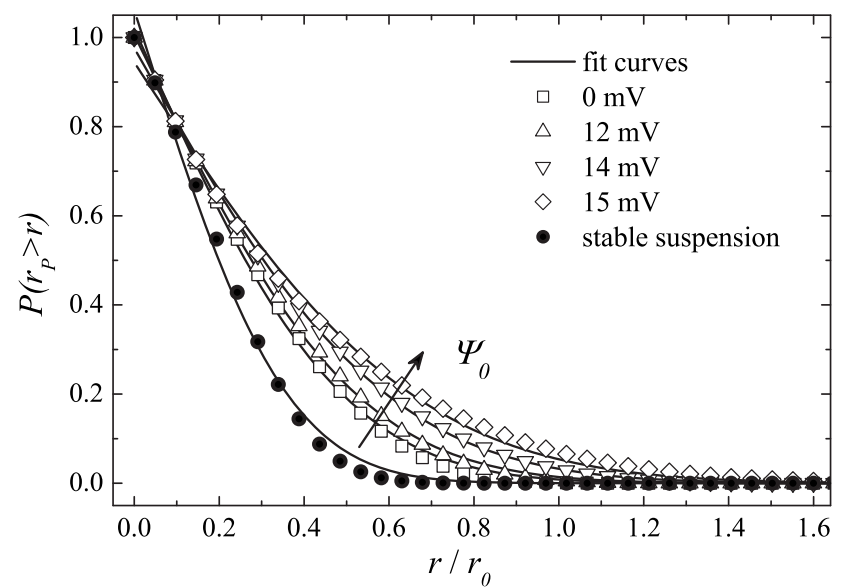

FIG. 4. Probability of finding pores with a radius $r_{P}$ larger than $r$ vs $r$ normalized by the particle radius $r_{0}$ for the various microstructures (symbols) and corresponding fits using a complementary error function (solid lines).

Toward larger pore radii, the probability ratio increases: finding pores with a radius larger than $0.75 r_{0}$ and $1.0 r_{0}$ is 5.1 and 60 times, respectively, more probable in the heterogeneous than in the homogeneous microstructure. Figure 4 further shows the fit of $P\left(r_{P}>r\right)$ using a complementary error function given by

$$
P\left(r_{P}>r\right)=1-\operatorname{erf}\left(\frac{r / r_{0}-b}{a \sqrt{2}}\right) .
$$

The error function is defined as the cumulative Gaussian distribution $\operatorname{erf}(x)=2 / \sqrt{\pi} \int_{0}^{x} \exp \left(-z^{2}\right) d z$. Parameter $a$ is the standard deviation, i.e., the width of the corresponding Gaussian distribution and $b$ is the location of its maximum, i.e., the most probable pore to particle radius ratio.

Table II summarizes the fit parameter $a$ and $b$ obtained for the various microstructures analyzed in this work and the corresponding correlation coefficients $R^{2}$, which for all fits are very close to 1 and thus indicate a good fit. Parameter $a$ is smallest for the initial microstructure and increases toward increasing values of $\Psi_{0}$. The increasing values of $a$ reflect the slower decrease of the curves in Fig. 4 and, hence, the broadening of the distributions toward increasing DOH. The values found for $b$ decrease with increasing $\Psi_{0}$ representing a shift of the maximum in the Gaussian distribution shown in Fig. 3 .
In [12], the following expression for $E_{V}(r)$ was found for a statistically homogeneous microstructure of impenetrable spheres $E_{V}(r)=(1-\Phi) \exp \left[P_{3}(r, \Phi)\right]$, where $\Phi$ is the volume fraction and $P_{3}$ is a third degree polynomial function in $r$. This function can be interpreted as a corrected Gaussian distribution, which is nicely approximated by Eq. (6) as well $\left(R^{2}=0.9962\right)$, yielding $a=0.347$ and $b=0.0204$. The DOH of this theoretical structure thus lies between the stable suspension and the most homogeneous final microstructure with $\Psi=0 \mathrm{mV}$.

An alternative to the quantification of a structure's heterogeneity by means of the fit parameter $a$ is the calculation of the integral over the cumulative pore size distribution. This scalar measure has the advantage of being statistically more robust. It is also more general in the sense that it is applicable even when the fit with a complementary error function does not yield good results. The integral over the cumulative pore size distribution is labeled $I_{p s}$ and is given by

$$
I_{p s}=\int_{r>0} P\left(r_{P}>r\right) \frac{d r}{r_{0}}=\frac{\delta r}{r_{0}} \sum_{r_{i}>0} P\left(r_{P}>r_{i}\right) .
$$

The second equality accounts for the discrete case, where $\delta r$ is the radial resolution of the empirical pore size distribution. The $I_{p s}$ values for the various microstructures are summarized in Table II. In our case, in which the data can nicely be fitted using Eq. (6), the fit parameter $a$ is proportional to $I_{p s}: a / I_{p s}=1.31 \pm 0.05$. Thus, a quantification of the DOH by means of $a$ or $I_{p s}$ is equivalent.

\section{B. Density fluctuations}

The density fluctuations for the various microstructures are shown in Fig. 5. Over the whole range of grid spacings, the fluctuations are smallest for the stable suspension and increase for increasing values $\Psi_{0}$. For $n_{c} \geq 34$, which corresponds to a grid spacing of 0.64 particle diameter, the density fluctuations of the various microstructures are equal. $I_{d f}$ given in Eq. (8) provides an integral measure of the heterogeneity similar to $I_{p s}$ in the previous section, however, accounting for the discrete variable $n_{c}$,

$$
I_{d f}=\sum_{n_{c}<34} \frac{\sigma_{p p c}}{E_{p p c}}\left(n_{c}\right) .
$$

The $I_{d f}$ values for the various microstructures summarized in Table II continuously increase toward increasing values of $\Psi_{0}$ and, thus, measure the DOH of the microstructures.

TABLE II. Fit parameters $a$ and $b, R^{2}$ values, and integrals $I_{p s}$ and $I_{d f}$ for the various microstructures.

\begin{tabular}{lccccc}
\hline \hline & $a$ & $b\left(10^{-2}\right)$ & $R^{2}$ & $I_{p s}$ & $I_{d f}$ \\
\hline Stable suspension & 0.2651 & 2.042 & 0.9952 & 0.215 & 20.29 \\
$\Psi_{0}=0 \mathrm{mV}$ & 0.3752 & 0.9013 & 0.9987 & 0.291 & 22.01 \\
$\Psi_{0}=12 \mathrm{mV}$ & 0.4053 & 0.3136 & 0.9994 & 0.311 & 22.31 \\
$\Psi_{0}=13 \mathrm{mV}$ & 0.4127 & 0.1225 & 0.9996 & 0.315 & 22.44 \\
$\Psi_{0}=14 \mathrm{mV}$ & 0.4736 & -1.415 & 0.9996 & 0.352 & 23.05 \\
$\Psi_{0}=15 \mathrm{mV}$ & 0.5377 & -3.756 & 0.9972 & 0.388 & 23.71 \\
\hline \hline
\end{tabular}




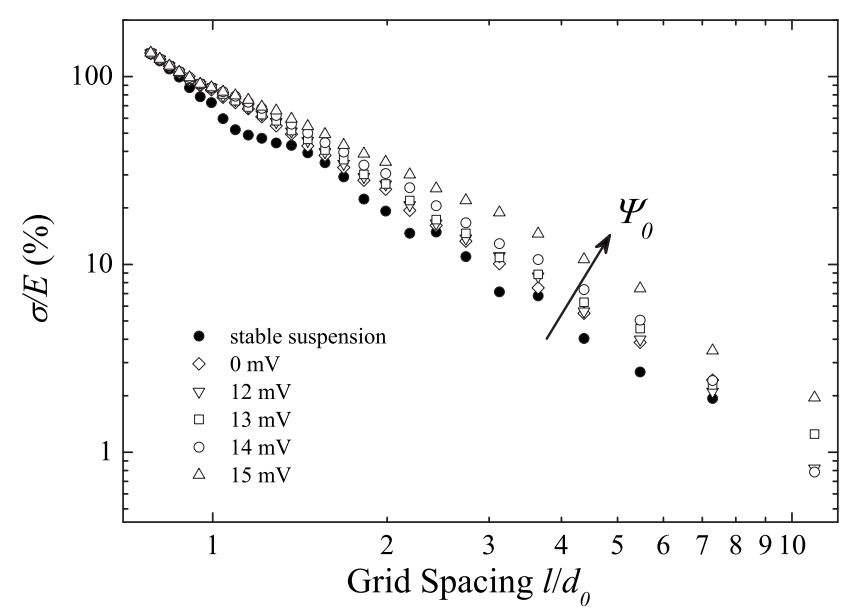

FIG. 5. Relative density fluctuations for the various final microstructures and the stable suspension as a function of grid spacing.

In the following, two sets of comparisons are performed. First, the various final microstructures are compared to the stable suspension. This comparison quantifies the length scale on which structural rearrangements take place during the coagulation. Second, the final microstructures with $\Psi_{0}$ $>0 \mathrm{mV}$ are compared to the most homogeneous microstructure with $\Psi_{0}=0 \mathrm{mV}$. This set of comparisons reveals the length scale on which variations in heterogeneity are most pronounced.

The comparison of the various final microstructures to the initial stabilized microstructure is shown in Fig. 6 in terms of $\Delta\left(n_{c}\right)$ as given by Eq. (4), where superscripts $i$ and $j$ correspond to a final microstructure and to the initial microstructure, respectively. $\Delta\left(n_{c}\right)$ is shown as a function of the grid spacing $l$ normalized by the particle diameter $d_{0}=2 r_{0}$.

All curves present an identical behavior, which essentially consists of three successive peaks with decreasing height toward a larger grid spacing. The location of the first, second, and third peak is slightly above one, at two, and at three particle diameters, respectively. The height of the individual peaks increases for increasing values $\Psi_{0}$.

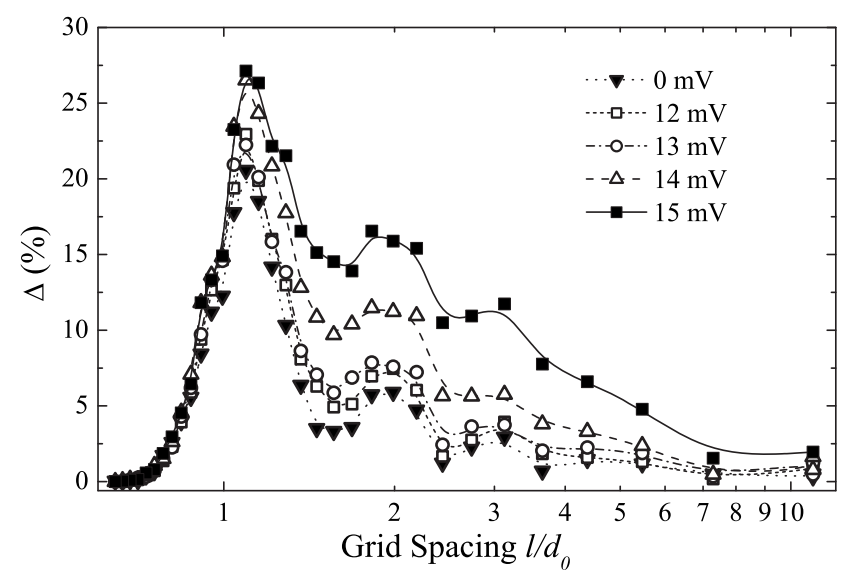

FIG. 6. Relative difference between the density fluctuations of the various final microstructures and the stable suspension as a function of grid spacing. $B$-spline curves serve as guide for the eyes.

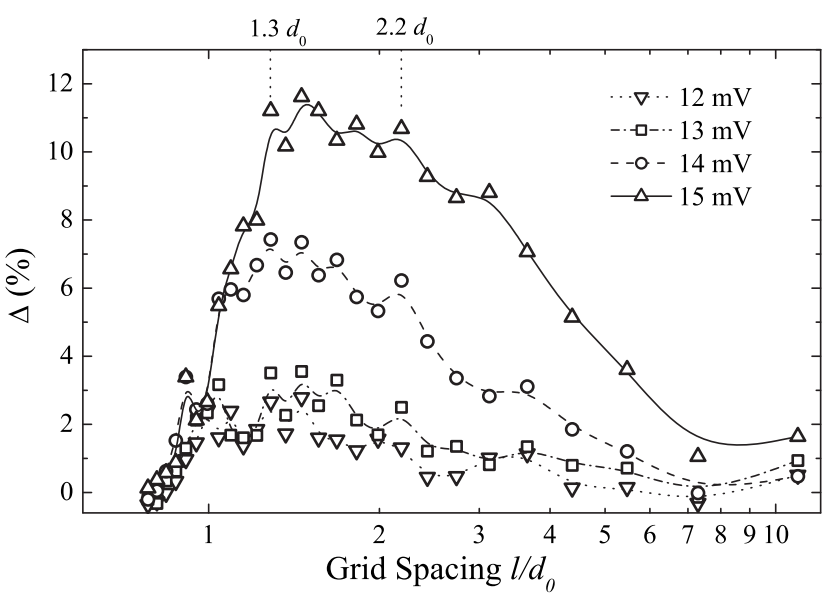

FIG. 7. Relative difference between the density fluctuations of the various microstructures with $\left(\Psi_{0}>0 \mathrm{mV}\right)$ and the most homogeneous microstructure $\left(\Psi_{0}=0 \mathrm{mV}\right)$ as a function of grid spacing. $B$-spline curves serve as guide for the eyes.

More precisely speaking, the first peak is found at $1.09 d_{0}$ for all final microstructures in comparison to the stable suspension. This grid spacing corresponds to a cell number of 8000 and, therefore, to the case where the average number of particles per cell is exactly one. This case is best reproduced for the stable suspension as for the final microstructures the standard deviation is roughly 20-27\% larger. Physically, this peak is explained by the transition of the interparticle potential from repulsive to attractive. Indeed, the repulsive potential in the case of the stable suspension causes all particles to occupy approximately the same volume as will be confirmed in Sec. III C by means of the Voronoi volume distribution. The switching of the interparticle potential from repulsive to attractive causes the particles to form contacts, resulting in an average particle separation of one particle diameter, which is smaller than the grid spacing of $1.09 d_{0}$. This increases the probability of finding cells that are either empty or contain more than one particle and, thus, the standard deviation of the average number of particles per cell is increased.

The peaks at a grid spacing of approximately two and three particle diameters are considerably less pronounced than the peak at $1.09 d_{0}$. In particular, the differences between the various final microstructures are larger than for the first peak. These differences will be elaborated in more detail in the following.

The comparison between the final microstructures with $\Psi_{0}>0 \mathrm{mV}$ and the most homogeneous microstructure with $\Psi_{0}=0 \mathrm{mV}$ is shown in Fig. 7. Here, superscripts $i$ and $j$ [Eq. (4)] correspond to one of the microstructures with $\Psi_{0}$ $>0 \mathrm{mV}$ and to the microstructure with $\Psi_{0}=0 \mathrm{mV}$, respectively. Over the whole range of grid spacings, the differences between the density fluctuations increase toward higher values of $\Psi_{0}$. This behavior correlates very well with the increase in porosity for increasing $\Psi_{0}$ as already observed in the previous section. Additionally, Fig. 7 reveals that the largest differences in terms or particle density between the most and least heterogeneous microstructures $\left(\Psi_{0}=15 \mathrm{mV}\right.$ and $\Psi_{0}=0 \mathrm{mV}$, respectively) are found on a length scale between 1.3 and 2.2 particle diameters. 


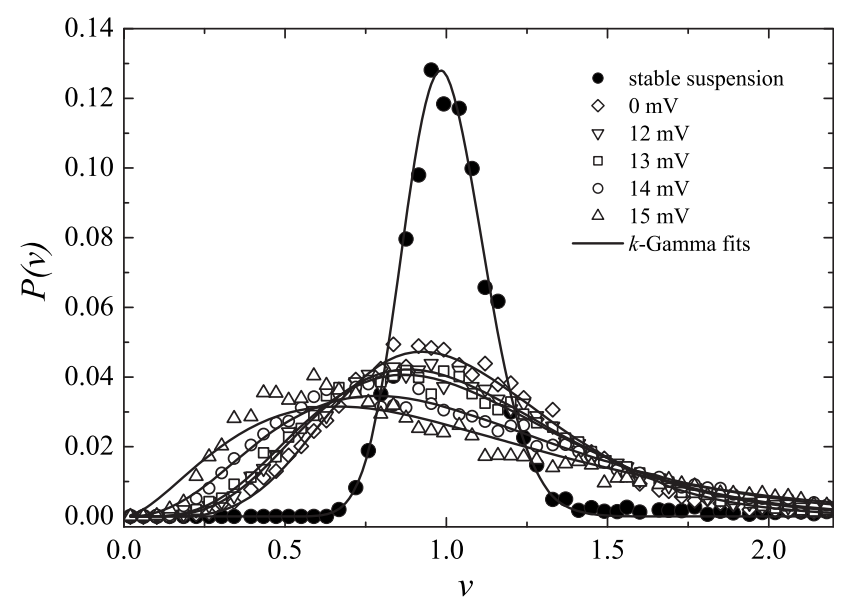

FIG. 8. Voronoi volume distribution $P(\nu)$ for the various microstructures (symbols) and corresponding fits using the $k$-gamma distribution (lines).

\section{Voronoi volume distribution}

As stated in Sec. II C, the distribution of Voronoi volumes $P(\nu)$ describes the deviation of a given structure from a perfectly crystalline packing, for which $P(\nu)$ is a delta function and all particles occupy the same volume. For random particle structures, $P(\nu)$ broadens and, as will be shown in the following, the width of the distribution can be interpreted as the heterogeneity of a structure.

We have calculated $P(\nu)$ for the stable suspension and the various final microstructures as a function of $\nu=\frac{V-V_{\min }}{\bar{V}-V_{\text {min }}}$, the free volume normalized by the mean free volume [Fig. 8 (symbols)]. The distribution found for the stable suspension is significantly narrower in comparison to the final microstructures. In the case of the final microstructures, $P(\nu)$ broadens with increasing value of $\Psi_{0}$ indicating that larger fluctuations in Voronoi volumes are found with increasing heterogeneity.

The various microstructures were fitted using the $k$-gamma distribution given in Eq. (5). The resulting curves are shown in Fig. 8 (lines) and the corresponding $k$ and $R^{2}$ values are summarized in Table III. $k$ decreases with increasing heterogeneity and can therefore be used as a measure for the DOH of the microstructures. The $R^{2}$ values close to one indicate good fits. In particular, a very good fit quality was achieved for the stable and the final microstructures up to $\Psi_{0}=14 \mathrm{mV}$. The $R^{2}$ value for the $\Psi_{0}=15 \mathrm{mV}$ microstruc-

TABLE III. $k$-gamma fit results.

\begin{tabular}{lrc}
\hline \hline & $k$ & $R^{2}$ \\
\hline Stable suspension & 62.3 & 0.990 \\
$\Psi_{0}=0 \mathrm{mV}$ & 8.6 & 0.995 \\
$\Psi_{0}=12 \mathrm{mV}$ & 6.5 & 0.996 \\
$\Psi_{0}=13 \mathrm{mV}$ & 6.0 & 0.996 \\
$\Psi_{0}=14 \mathrm{mV}$ & 4.0 & 0.994 \\
$\Psi_{0}=15 \mathrm{mV}$ & 2.8 & 0.972 \\
\hline \hline
\end{tabular}

ture is lower. Indeed, Fig. 8 shows that for the $\Psi_{0}=15 \mathrm{mV}$ microstructure, the agreement between the measured distribution and the fit curve for small Voronoi volumes is not as good as for the other curves. This might be related to the fact that during the generation of the $\Psi_{0}=15 \mathrm{mV}$ microstructure, the energy barrier between primary and secondary minimum in the interparticle potential was largest. This resulted in a few particle contacts still trapped in the secondary minimum (roughly $7 \%$ of the physical contacts). Particles trapped in the secondary minimum have an interparticle distance of $2.16 r_{0}$ instead of $2 r_{0}$ upon complete coagulation, which may be a reason for the reduced fit quality toward smaller Voronoi volumes.

\section{SUMMARY AND CONCLUSIONS}

In this paper, we have analyzed three distinct microstructural characterization methods. Using these methods, scalar measures were introduced, which allow quantifying the DOH of particle packings.

(i) The exclusion probability gives an estimate of the pore size distribution by a random probing of the pore space. The more heterogeneous microstructures present a considerably broader pore size distribution with a significantly longer tail than the distribution for the more homogeneous microstructures. In particular, a continuous broadening is found with increasing heterogeneity. The cumulative exclusion probabilities were shown to follow error functions with parameter $a$ reflecting their width and thus measuring the structures' DOH. Fit parameter $a$ increases with increasing heterogeneity.

(ii) The density-fluctuation method statistically analyzes the particle center density in dependence of the sampling length scale. The relative density fluctuation as function of the grid spacing presents a clear dependence on the heterogeneity of the microstructure. Over the whole range of grid spacings, the stable suspension exhibits the smallest density fluctuations. These fluctuations increase toward increasing values of $\Psi_{0}$ and thus increasing $\mathrm{DOH}$, which is nicely reflected by increasing values of the integral measure $I_{d f}$.

An examination of the differences between the density fluctuations of two structures was found to be particularly useful as it allows determining the length scale on which the structures present the largest differences in heterogeneity. In the case of the most heterogeneous microstructure, the largest differences in comparison to the most homogeneous one are found on a length scale between 1.3 and 2.2 particle diameters.

(iii) The Voronoi volume distribution of the stable suspension is very narrow in comparison to the final microstructures, for which the distribution broadens with increasing heterogeneity. The various Voronoi volume curves were shown to follow $k$-gamma distributions. Parameter $k$, reflecting the width of the distribution and thus the structure's $\mathrm{DOH}$, decreases with increasing heterogeneity.

The behavior of the three parameters $a, k$, and $I_{d f}$ is summarized in Fig. 9 showing $I_{d f}$ (left scale) and $1 / k$ (right scale) as a function of $a$. The solid lines suggest a pairwise affine dependence between $I_{d f}$ and $1 / k, a$. Thus, as far as the 


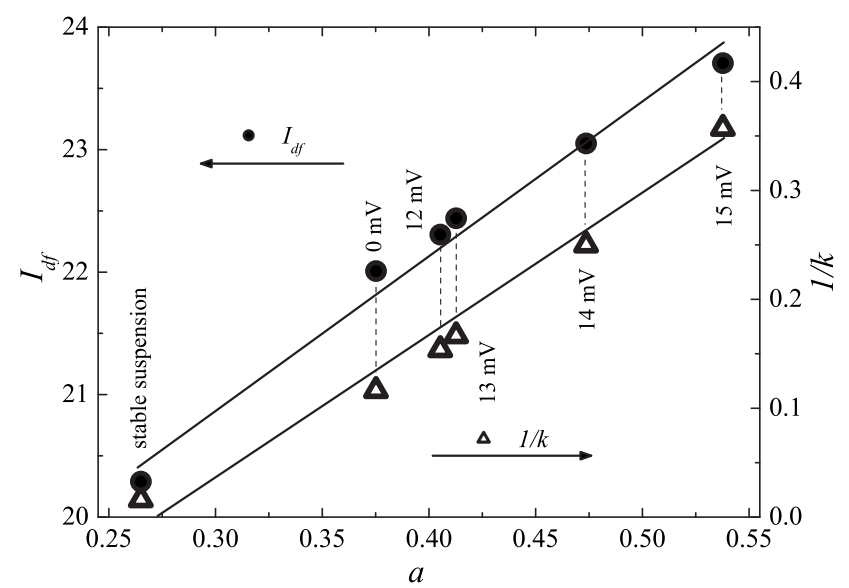

FIG. 9. Interdependence between the measures of the degree of heterogeneity for the various microstructures: $I_{d f}$ and $1 / k$ as a function of $a$.

quantitative characterization of the DOH of the microstructures considered in this work is concerned, all methods-the pore size distribution, the density-fluctuation method, and the Voronoi volume distribution-can be considered as equivalent in the sense that the knowledge of one parameter permits to determine the others. However, parameter $k$ reflects changes in the DOH more sensitively than $a$ or $I_{d f}$. Indeed, the normalization of $k, a$, and $I_{d f}$ with respect to their maximum values reveals that parameter $k$ covers the interval $[0.04,1.0]$. This interval is significantly larger than the normalized ranges of $a$ and $I_{d f}$, which are $[0.5,1.0]$ and $[0.86$, 1.0], respectively.

The interrelation between the three structural characterization methods can be understood as follows. The probability of placing a random point used for the determination of the pore size distribution into the free Voronoi volume of a particle is proportional to the particle's free Voronoi volume. Thus, the broader the distribution of Voronoi volumes, the higher the probability of finding larger pores, which leads to a longer tail in the exclusion probability as shown in Fig. 3. The relation between the Voronoi volume distribution and the density fluctuation follows similar arguments. A broadening in the Voronoi volume distribution essentially means that there is a broader distribution in the nearest-neighbor distances and therefore larger differences in the density fluctuations.

We have applied the methods to a set of monodispersed spherical particle packings representing stable and coagulated colloidal particle structures, but the methods could of course be generalized. The pore size distribution as determined by the Monte Carlo method employed in this work can be applied as it is to any porous media. In this sense, it is the most general method analyzed in this study. The fit using an error function, however, may not necessarily yield good results. In this case, the integral measure $I_{p s}$ proposed in Sec. III A could be used or the width of the distribution could be determined empirically. The Voronoi volume distribution generally only requires that the elements constituting a structure are convex and, in this case, the empirical distribution can be determined. To the authors' knowledge, a fit using the $k$-gamma distribution has, however, only been performed in the case of packings of monodispersed spherical particles. As for the density-fluctuation method, we have in this work considered the density of the particle centers. The method may be extended to a determination of the exact portion of the sphere volumes per cell, which, however, is computationally expensive. An alternative could be a cellwise Monte Carlo integration of the partial sphere volumes, which would allow for a characterization of arbitrary porous structures using the density-fluctuation method.

From an experimental point of view, the methods presented in this study rely on the possibility to determine the particle positions, which in the case of colloidal particles can be obtained using the confocal laser microscopy, for example [24]. In particular, the pore size distributions measured using mercury porosimetry [25] and estimated using the exclusion probability are not equivalent since the latter overestimates the number of small pores due to the random probing of the pore space.

In this paper, we have presented three scalar measures, which allow quantifying and comparing the heterogeneity of packings of spherical particles in terms of a DOH. These measures were calculated using distinct techniques and structural characterization methods. In view of these differences, the very nice correlation between the three $\mathrm{DOH}$ measures is remarkable. Indeed, it suggests that the $\mathrm{DOH}$ is a microstructure's inherent property and that any of the methods proposed in this work can be used to uniquely characterize and classify it. In terms of sensitivity, however, considerable differences between the methods were found. Parameter $k$ reflects differences in the DOH most sensitively, followed by parameter $a$, and finally by $I_{d f}$. A further definition of an absolute DOH would require a suitable reference structure, which, for example, is either perfectly heterogeneous or perfectly homogeneous under the condition of being random.

\section{ACKNOWLEDGMENTS}

The authors would like to express their gratitude to Markus Hütter for providing the colloidal microstructure data from the Brownian dynamics simulations. Additionally, Iwan Schenker would like to thank Henning Galinski and Joakim Reuteler for helpful discussions. 
[1] J. Visser, Powder Technol. 58, 1 (1989).

[2] J. N. Israelachvili, Intermolecular \& Surface Forces, 2nd ed. (Academic Press, London, 1991).

[3] L. J. Gauckler, T. Graule, and F. Baader, Mater. Chem. Phys. 61, 78 (1999).

[4] E. Tervoort, T. A. Tervoort, and L. J. Gauckler, J. Am. Ceram. Soc. 87, 1530 (2004).

[5] H. M. Wyss, S. Romer, F. Scheffold, P. Schurtenberger, and L. J. Gauckler, J. Colloid Interface Sci. 241, 89 (2001).

[6] H. M. Wyss, M. Hütter, M. Müller, L. P. Meier, and L. J. Gauckler, J. Colloid Interface Sci. 248, 340 (2002).

[7] M. Hütter, J. Colloid Interface Sci. 231, 337 (2000).

[8] M. Hütter, Ph.D. thesis, ETH Zurich, Switzerland, 1999; http:// e-collection.ethbib.ethz.ch/show?type $=$ diss $\& n r=13107$

[9] M. Hütter, Phys. Rev. E 68, 031404 (2003).

[10] R. Andersson, L. F. van Heijkamp, I. M. de Schepper, and W. G. Bouwman, J. Appl. Crystallogr. 41, 868 (2008).

[11] I. Schenker, F. T. Filser, T. Aste, and L. J. Gauckler, J. Eur. Ceram. Soc. 28, 1443 (2008).

[12] S. Torquato, B. Lu, and J. Rubinstein, Phys. Rev. A 41, 2059 (1990).

[13] M. Whittle and E. Dickinson, Mol. Phys. 96, 259 (1999).
[14] A. Bezrukov, M. Bargieł, and D. Stoyan, Part. Part. Syst. Charact. 19, 111 (2002).

[15] G. Voronoi, J. Reine Angew. Math. 134, 198 (1908).

[16] R. Y. Yang, R. P. Zou, and A. B. Yu, Phys. Rev. E 65, 041302 (2002).

[17] P. A. Cundall and O. D. L. Strack, Geotechnique 29, 47 (1979).

[18] T. Aste and T. Di Matteo, Phys. Rev. E 77, 021309 (2008).

[19] W. B. Russel, D. A. Saville, and W. R. Schowalter, Colloidal Dispersions (Cambridge University Press, New York, 1989).

[20] H. M. Wyss, A. M. Deliormanli, E. Tervoort, and L. J. Gauckler, AIChE J. 51, 134 (2005).

[21] C. B. Barber, D. P. Dobkin, and H. Huhdanpaa, ACM Trans. Math. Softw. 22, 469 (1996).

[22] V. Senthil Kumar and V. Kumaran, J. Chem. Phys. 123, 114501 (2005).

[23] M. Matsumoto and T. Nishimura, ACM Trans. Model. Comput. Simul. 8, 3 (1998)

[24] J. C. Crocker and D. G. Grier, J. Colloid Interface Sci. 179, 298 (1996).

[25] H. Giesche, Part. Part. Syst. Charact. 23, 9 (2006). 